Article

\title{
Evaluate the Differences in CT Features and Serum IgG4 Levels between Lymphoma and Immunoglobulin G4-Related Disease of the Orbit
}

\author{
Wei-Hsin Yuan ${ }^{1,2,3, *(D)}$, Anna Fen-Yau Li ${ }^{3,4}$, Shu-Yi Yu ${ }^{2,3}$, Ying-Yuan Chen ${ }^{3,5}$, Chia-Hung Wu ${ }^{2,3}$, \\ Hui-Chen $\mathrm{Hsu}^{6}{ }^{6}$, Jiing-Feng Lirng ${ }^{2,3}$ and Wan-You Guo ${ }^{2,3}$ \\ 1 Division of Radiology, Taipei Municipal Gan-Dau Hospital (Managed by Taipei Veterans General Hospital), \\ Taipei 11260, Taiwan \\ 2 Department of Radiology, Taipei Veterans General Hospital, Taipei 11217, Taiwan; \\ frankfbo@gmail.com (S.-Y.Y.); chwu16@vghtpe.gov.tw (C.-H.W.); jflirng@vghtpe.gov.tw (J.-F.L.); \\ wyguo@vghtpe.gov.tw (W.-Y.G.) \\ 3 School of Medicine, National Yang-Ming University, Taipei 10556, Taiwan; Fyli@vghtpe.gov.tw (A.F.-Y.L.); \\ yychen354@gmail.com (Y.-Y.C.) \\ 4 Department of Pathology, Taipei Veterans General Hospital, Taipei 11217, Taiwan \\ 5 Division of Radiology, National Yang-Ming University Hospital, Yilan City 26058, Taiwan \\ 6 Department of Medical Imaging, Taiwan Adventist Hospital, Taipei 10556, Taiwan; hueichenhsu@gmail.com \\ * Correspondence: williamyuan.tw@gmail.com
}

Received: 14 June 2020; Accepted: 27 July 2020; Published: 29 July 2020

check for updates

\begin{abstract}
Background: Benign immunoglobulin G4 (IgG4)-related orbital disease (IgG4-ROD) characterized as tumors mimicking malignant orbital lymphoma (OL) — responds well to steroids, instead of chemotherapy, radiotherapy and/or surgery of OL. The objective of this study was to report the differences in computed tomography (CT) features and- serum IgG4 levels of IgG4-ROD and OL. Methods: This study retrieved records for patients with OL and IgG4-ROD from a pathology database during an eight-year-and-five-month period. We assessed the differences between $16 \mathrm{OL}$ patients with 27 lesions and nine IgG4-ROD patients with 20 lesions according to prebiopsy CT features of lesions and prebiopsy serum IgG4 levels and immunoglobulin G (IgG) levels This study also established the receiver-operating curves (ROC) of precontrast and postcontrast CT Hounsfield unit scales (CTHU), serum IgG4 levels, serum IgG levels and their ratios. Results: Significantly related to IgG4-ROD (all $p<0.05$ ) were the presence of lesions with regular borders, presence of multiple lesions-involving both lacrimal glands on CT scans-higher median values of postcontrast CTHU, postcontrast CTHU/precontrast CTHU ratios, serum IgG4 levels and serum IgG4/IgG level ratios. Compared to postcontrast CTHU, serum IgG4 levels had a larger area under the ROC curve ( 0.847 [ $95 \%$ confidence interval (CI): $0.674-1.000, p=0.005$ ] vs. 0.766 [95\% CI: $0.615-0.917, p=0.002]$ ), higher sensitivity $(0.889$ [95\% CI: $0.518-0.997]$ vs. 0.75 [95\% CI: $0.509-0.913])$, higher specificity (0.813 [95\% CI: $0.544-0.960$ ] vs. 0.778 [95\% CI: 0.578-0.914]) and a higher cutoff value ( $\geq 132.5 \mathrm{mg} / \mathrm{dL}$ [milligrams per deciliter] vs. $\geq 89.5$ ). Conclusions: IgG4-ROD showed distinct CT features and elevated serum IgG4 ( $\geq 132.5 \mathrm{mg} / \mathrm{dL}$ ), which could help distinguish IgG4-ROD from OL.
\end{abstract}

Keywords: immunoglobulin G4-related orbital disease (IgG4-ROD); orbital lymphoma (OL); computed tomography (CT); Hounsfield unit

\section{Introduction}

Orbital space-occupying lesions comprise a wide range of benign and malignant masses [1]. The top eight ocular adnexal lesions include lymphoid tumors, inflammatory disease, cavernous 
hemangioma, lymphangioma, meningioma, optic nerve glioma, metastatic breast cancer and capillary hemangioma [2]. Several studies indicate that ocular adnexal lymphomas almost account for up to half of all malignant orbital lesions in adults [2-4].

Immunoglobulin G4-related disease-which can involve any organs including orbital structuresis a systemic fibroinflammatory condition due to tissue infiltration by immunoglobulin G4 (IgG4) plasma cells [5]. IgG4-related disease in orbit tends to form tumor-like lesions, which are difficult to differentiate from intraorbital lymphoma because both are tumors rich in lymphoplasmacytic infiltration [6]. Lymphoma, one of the most common orbital malignancies in adults [3,4], needs radiotherapy, systemic chemotherapy and/or surgery $[7,8]$. In contrast, approximately $90 \%$ of patients with IgG4-related orbital disease (IgG4-ROD) respond well to steroid therapy [5] Therefore, rapid and accurate diagnosis of IgG4-ROD to help patients receive early steroid treatment is critical.

IgG4-related disease diagnostic criteria commonly follow: a serum IgG4 concentration higher than $135 \mathrm{mg} / \mathrm{dL}$ (milligrams per deciliter) [9], the ratio of IgG4-positive/immunoglobulin G (IgG)-positive plasma cell (IgG4+/IgG+ ratio) is $>40 \%$ or IgG4 + cells $>10 /$ high-powered field of biopsy sample [9]. However, up to $40 \%$ of patients with IgG4-related disease may have serum IgG4 levels within the normal range [10]. Pathology and immunohistochemistry remain the gold standard for accurate diagnosis of IgG4-related disease [5,11].

Furthermore, orbital neoplasm rupture via biopsy may lead to tumor seeding and poor prognosis [12]. Integrating clinical findings, serologic data and radiological features is important to establish the prebiopsy diagnosis of IgG4-ROD [12]. Computed tomography (CT) scans provide rapid high-resolution images of orbits for radiological feature extraction [13]. As such, this study integrates CT qualitative and quantitative (Hounsfield unit density) features, serum IgG, and IgG4 levels to differentiate IgG4-ROD from orbital lymphoma (OL).

\section{Material and Methods}

\subsection{Patients}

The Institutional Review Board of Taipei Veterans General Hospital (TVGH) approved this study to waive informed consent because of the retrospective nature of the research.

A doctor (SYY) blinded from the research hypothesis searched pathologic results from the pathology database at TVGH using the keywords "orbit" or "orbital" for cases from 1 January 2010, to 31 May 2018.

The research returned 178 patients with orbital lesions and pathologic results. Thirteen $(7 \%)$ of 178 patients had multiple orbital lesions $(\geq 2)$ : one $(7.7 \%)$ of the thirteen patients with lung carcinoid tumors and multiple metastases in the right orbital cavity and $12(92.3 \%)$ patients with lymphoplasmacyte-rich lesions (5 patients with orbital lymphoma; 7 patients with IgG4-ROD). The other $165(93 \%)$ of the 178 patients showed only one lesion in the orbital cavity or eyelid. Sixteen $(9 \%)$ of the 178 patients had orbital lymphoma (OL) and $9(5 \%)$ patients had IgG4-related orbital disease (IgG4-ROD).

Among these patients, this study only considered patients who had OL or IgG4-ROD with prebiopsy precontrast and postcontrast orbital CT scans, serum IgG4 levels and serum IgG levels and excluded those were younger than 20 years of age or pregnant cases or those lacked prebiopsy CT and serologic data.

As a result, 25 patients pathologically diagnosed as OL (16 patients) or IgG4-ROD (9 patients) met the inclusion criteria and had prebiopsy orbital CT scans, serum IgG4 levels and serum IgG levels. All patients met the eligibility criteria. We enrolled 25 patients to collect and analyze demographic data, symptoms and signs, past medical histories, CT qualitative and quantitative (Hounsfield unit density) features of orbital lesions, serum IgG levels and serum IgG4 levels of patients with IgG4-ROD from those of patients with OL. 


\subsection{CT Imaging Techniques}

This study examined orbital CT images taken by a multiple-detector computed tomography (MDCT) scanner for the selected 25 patients. MDCT scanners of orbit or face included iCT 256 (256-slice, $n=5)$, Philips Healthcare, Cleveland, OH, USA, Somatom Sensation 16 (16-slice, $n=4)$, Siemens Healthcare, Forchheim, Germany, ECLOS Hitachi Medical Corporation (16-slice, $n=1$ ), Tokyo, Japan, and Aquilion 64, Toshiba Medical Systems (64-slice, $n=15$ ), Tochigi, Japan. Orbital CT scans were obtained with or without an intravenous contrast medium, which included iobitridol (Xenetix 350; Guerbet, Rue Jean Chaptal, Aulnary-sous-Bios, France, $350 \mathrm{mg}$ I [Iodine]/mL [milliliter]) and iohexol (Omnipaque 350; GE healthcare, Carrigtohill, Co., Cork, Ireland, $350 \mathrm{mg} \mathrm{I} / \mathrm{mL}$ ). The data records showed that twenty-five patients underwent an intravenous power injection as a bolus of $1.2-\mathrm{mL} / \mathrm{kg}$ (kilogram) iodine-based contrast medium at $1 \mathrm{~mL} /$ second (s). Postcontrast CT images were performed after the complete injection of contrast medium. The axial sections of precontrast and postcontrast orbital CT images scanned along the transaxial direction with the sections parallel to the optic nerve along a line from the inferior border of the maxillary sinus to the middle part of the frontal region. A Hitachi CT scanner took the slice thickness for image viewing of axial images at $1.25 \mathrm{~mm}(\mathrm{~mm})$ and other MDCT scanners at 2-4 mm. The coronal and sagittal sections of postcontrast orbital CT images were reformatted with 2-4 mm in slice thickness. The reconstruction matrix for MDCT scans of orbit was $512 \times 512$.

\subsection{Analysis of Images and Pathologic Diagnosis}

Two experienced radiologists (CHW and YYC) reanalyzed orbital lesions of the 25 patients on orbital CT images with axial, sagittal and/or coronal images together without knowledge of pathologic diagnosis of orbital tumors. The consensus from the two radiologists served as the final interpretation of images. If the two radiologists could not reach an agreement on any features from orbital CT scans, a third experienced radiologist $(\mathrm{HCH})$ mediated the disagreement.

This study analyzed the following orbital CT features of each lesion or of each patient: maximum diameter of a lesion, lesion borders, homogeneity of CT density, a lesion involving extraocular muscle tendons, the lacrimal sac, lacrimal gland, preseptal space, extraconal, conal or intraconal orbital compartments, the optic nerve, infraorbital nerve, presence or absence of bone remodeling, single tumor or multiple lesions and single or bilateral orbital involvement, single or bilateral lacrimal gland involvement. This study also measured the mean values of the precontrast and postcontrast CT Hounsfield unit scales (CTHU) of each orbital lesion among the 25 patients.

A regular border of an orbital tumor on CT scans indicated the contour of a lesion from the surrounding tissue was smooth for more than $75 \%$ of the lesion. An irregular border of a lesion showed microlobulated, microangulated or indistinct contour from the surrounding tissue with $\geq 25 \%$ of the lesion. Lesion involvement indicated lesion infiltration, invasion or encasement on orbital CT scans.

This study measured CTHU for all 47 orbital lesions of 25 patients on both pre and postcontrast prebiopsy CT scans. The region of interest (ROI) maker in an oval shape was placed in the center of each lesion to cover $50 \%$ of the largest tumor area on $\mathrm{CT}$ axial images, avoiding the inclusion of bone and blood vessels (Figure 1). This study also calculated postcontrast CTHU divided by precontrast CTHU. 


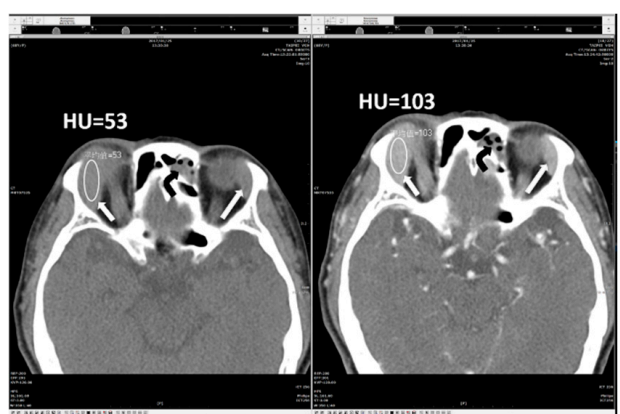

(a)

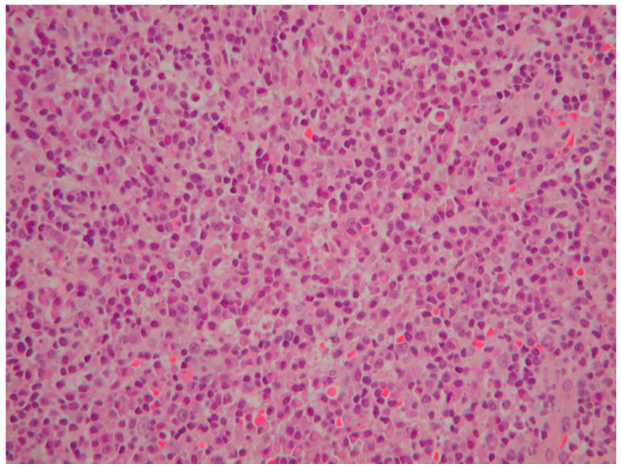

(c)

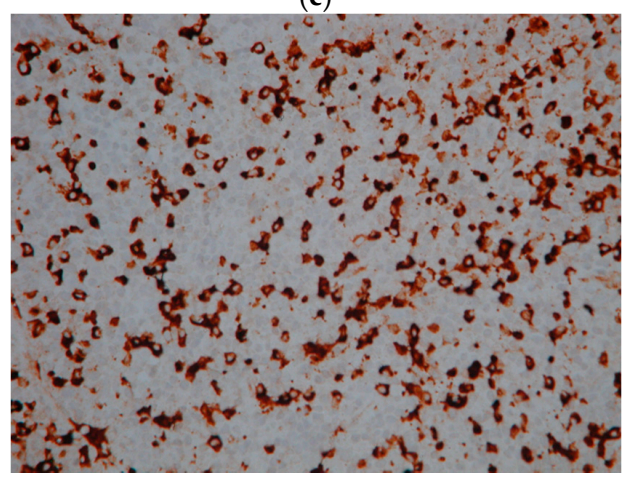

(e)

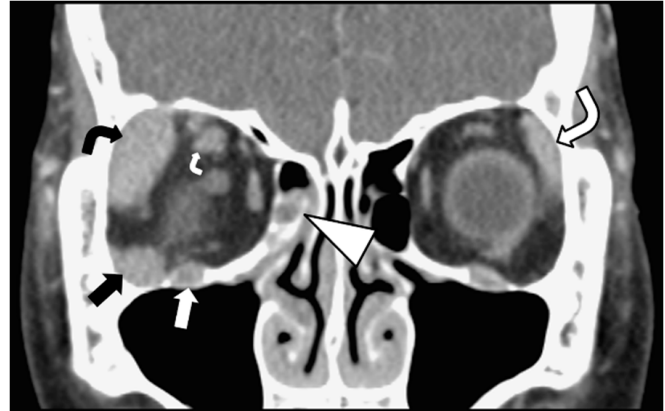

(b)

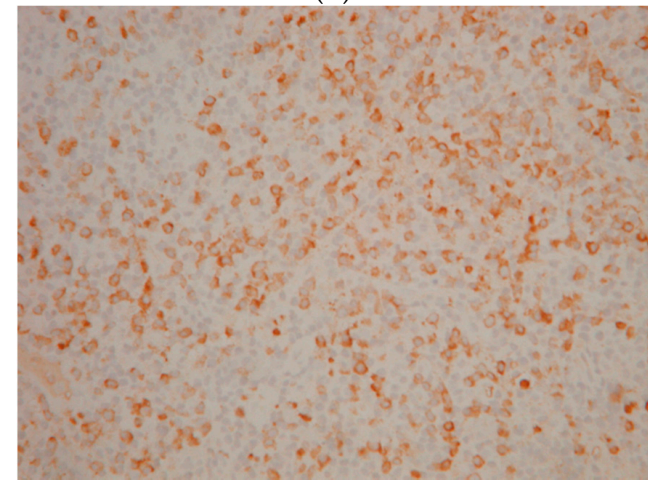

(d)

Figure 1. A 68-year-old man with immunoglobulin G4 (IgG4)-related orbital disease (IgG4-ROD) shows multiple tumors in bilateral orbital cavities. (a) Axial computed tomography (CT) scans show masses in bilateral lacrimal glands (short and large arrows). The mean value of CT Hounsfield unit scale (CTHU) is measured at the enlarged right lacrimal gland (short arrows) on a picture archiving and communication system monitor. The region of interest (ROI) marker in an oval shape is placed in the center of the mass (short arrows) to cover $50 \%$ of the largest tumor area. The mean value of precontrast CTHU is 53 and that of postcontrast CTHU is 103. Sinusitis is found in the left frontal sinus with mucus retention (black curved arrows); (b) Coronal postcontrast CT scan shows multiple masses or enlargement in various ophthalmic tissues with regular borders and homogeneous contrast enhancement in bilateral orbital cavities as follows: a mass at the extraconal compartment of the right orbital cavity (black arrow), the right lacrimal gland (black curved arrow), the left lacrimal gland (large white curve arrow), the right superior rectus muscle belly (small white curve arrow) and the right infraorbital nerve (white arrow). Sinusitis is noted in the right ethmoid sinus with mucus retention (arrowhead); (c) Pathologic specimen shows infiltration of many lymphoplasma cells and mild fibrosis (hematoxylin-eosin stain, original magnification $\times 200$ ); (d) Immunostaining for immunoglobulin $G$ (IgG)-expression shows many plasma cells are positive for IgG stains (original magnification $\times 200$ ); (e) Immunostaining for IgG4-expression shows abundant IgG4-positive plasma cells have infiltrated the lesion. IgG4-postive/IgG-positive plasma cell ratio is more than $40 \%$. There are more than 100 IgG4-positive plasma cells in one high-powered field (>100/HPF) (original magnification $\times 200$ ). 
An experienced pathologist (AFYL) with 29 years of experience in pathology diagnosis reviewed the pathologic and immunohistochemical sections of the specimens of the 25 patients to confirm pathologic results of OL and IgG4-ROD. The two main pathologic criteria of IgG4-ROD included (1) IgG4+/IgG+ ratio > 40\%, and/or (2) IgG4+ cells > 10/high-powered field (HPF) in histopathologic examination $[5,9,14]$.

The radiologist (WHY) integrated demographic data, patient symptoms, signs and past histories, prebiopsy serum IgG4 levels and IgG levels and CT imaging interpretations and the mean values of CTHU measurement results of the 25 patients to evaluate the differences in $\mathrm{CT}$ qualitative and quantitative features, serum IgG and IgG4 levels between OL and IgG4-ROD.

\subsection{Statistical Analysis}

This study used SPSS version 19.0 software (SPSS, Inc., Chicago, IL, USA) for data analysis. Specially, we applied the Mann-Whitney U test to compare continuous variables because of the small sample size and the $\chi 2$ or Fisher's exact test for categorical variables at the level of significance of $p<0.05$. Receiver operating characteristic (ROC) curve analysis calculated the area under the ROC curve to identify diagnostic values of CTHU, serum IgG4 levels and serum IgG levels of IgG4-ROD. This study assessed the findings based on sensitivity, specificity and accuracy with a 95\% confidence interval $(95 \% \mathrm{CI})$.

\section{Results}

The median age (mean \pm standard deviation [SD], range) of the selected 25 patients was 59 $(58.20 \pm 10.61,32-78)$. The median age (mean \pm SD, range) of 16 patients with OL was $60.5(59.31 \pm 9.20$, $41-78)$ and that of 9 patients with IgG4-ROD was $58(56.22 \pm 13.11,32-69)(p=0.934$, Mann-Whitney $\mathrm{U}$ test). Of the 25 patients, $17(68 \%)$ were male and $8(32 \%)$ were female. Twelve ( 12 or $71 \%)$ of the 17 male patients were OL and $5(29 \%)$ were IgG4-ROD; four (50\%) of 8 females were OL patients and $4(50 \%)$ were IgG4-ROD ( $p=0.3942$, Fisher's exact test).

The 25 patients showed proptosis, palpable mass and/or eyelid swelling-none of the 25 patients suffered from orbital pain or tender palpable mass. Six ( 6 or $24 \%$ ) of the 25 patients had malignancy histories. Five (83\%) of the 6 patients with malignant histories had OL: one with renal cell carcinoma, one with prostatic cancer and soft palate follicular lymphoma, one with squamous cell carcinoma of the tongue, one with follicular lymphoma involving lung, neck lymph nodes and bone marrow and one with chronic lymphocytic leukemia. Only one $(17 \%)$ of the 6 patients with malignant history was an IgG4-ROD patient who had ovarian cancer. Patient malignant histories of the two groups had no significant difference ( $p=0.3644$, Fisher's exact test).

A pathologist (AFYL) reviewed the pathologic sections of the 25 patients. The pathologic review concluded 13 patients with extranodal marginal zone lymphoma of mucosa-associated lymphoid tissue (MALT lymphoma), 1 with low-grade B cell lymphoma with plasmacytic differentiation, 1 with diffuse large B cell lymphoma, 1 with follicular lymphoma and 9 with IgG4-ROD. The histopathologic findings of the 9 patients with IgG4-ROD showed diffuse lymphoplasmacytic infiltration, IgG4-positive (IgG4+) plasma cells, IgG-positive (IgG+) plasma cells and various degree fibrosis. Seven (78\%) of the nine IgG4-ROD patients showed IgG4+ cells $>100$ cells/HPF and IgG4+/IgG+ ratio > 40\% (Figure 1). Another 2 of the 9 IgG4-ROD patients (22\%) had IgG4+ plasma cell $<50$ cells/HPF and IgG4+/IgG+ ratio $>40 \%$.

Furthermore, CT images indicated a total of 47 orbital tumors among the 25 patients: 27 lesions were OL and 20 lesions were IgG4-ROD. Of 47 orbital tumors, none appeared inside the eyeball.

Tables 1 and 2 summarize CT features of 47 tumors among the 25 patients, of which 16 had orbital lymphoma and 9 had IgG4-ROD. 
Table 1. Computed tomography (CT) features of 47 tumors among the 25 patients with orbital lymphoma or immunoglobulin G4-related orbital disease (IgG4-ROD) on prebiopsy orbital CT scans.

\begin{tabular}{|c|c|c|c|}
\hline Orbital CT Scans & $\begin{array}{c}\text { Orbital Lymphoma } \\
n(\%)\end{array}$ & $\begin{array}{c}\text { IgG4-ROD } \\
n(\%)\end{array}$ & $p$ \\
\hline Tumor Size, median (mean $\pm S D$, range) & $2.58(2.62 \pm 1.149,0.98-5.16)$ & $3.17(2.70 \pm 1.233,0.66-5.1)$ & $0.667^{\circledR}$ \\
\hline Lesion border & & & $0.0069^{\#}$ \\
\hline Regular & $10(38)$ & $16(62)$ & \\
\hline Irregular & $17(81)$ & $4(19)$ & \\
\hline Precontrast CT density & & & $1^{\#}$ \\
\hline Homogeneous & $26(57)$ & $20(43)$ & \\
\hline Heterogeneous & $1(100)$ & $0(0)$ & \\
\hline Postcontrast CT contrast-enhancement & & & $1^{\#}$ \\
\hline Homogeneous & $26(57)$ & $20(43)$ & \\
\hline Heterogeneous & $1(100)$ & $0(0)$ & \\
\hline Extraocular muscle tendon involvement & & & $0.1138^{\#}$ \\
\hline Presence & $10(77)$ & $3(23)$ & \\
\hline Absence & $17(50)$ & $17(50)$ & \\
\hline Lacrimal sac involvement & & & $1^{\#}$ \\
\hline Presence & $3(60)$ & $2(40)$ & \\
\hline Absence & $24(57)$ & $18(43)$ & \\
\hline Preseptal space involvement & & & $1^{\#}$ \\
\hline Presence & $8(62)$ & $5(38)$ & \\
\hline Absence & $19(56)$ & $15(44)$ & \\
\hline Lacrimal gland involvement & & & $0.0085^{\#}$ \\
\hline Presence & $8(36)$ & $14(64)$ & \\
\hline Absence & $19(76)$ & $6(24)$ & \\
\hline Orbital compartment involvement & & & $0.4813^{\#}$ \\
\hline Extraconal or/and conal & $20(53)$ & $17(47)$ & \\
\hline Intraconal & $7(78)$ & $3(22)$ & \\
\hline Optic nerve involvement & & & $1^{\#}$ \\
\hline Presence & $5(63)$ & $3(37)$ & \\
\hline Absence & $22(56)$ & $17(44)$ & \\
\hline Infraorbital nerve involvement & & & $1^{\#}$ \\
\hline Presence & $1(50)$ & $1(50)$ & \\
\hline Absence & $26(58)$ & $19(42)$ & \\
\hline Bone remodeling & & & $0.2507^{\#}$ \\
\hline Presence & $3(100)$ & $0(0)$ & \\
\hline Absence & $24(55)$ & $20(45)$ & \\
\hline
\end{tabular}

Table 2. CT features of the 25 patients with orbital lymphoma or immunoglobulin G4-related orbital disease (IgG4-ROD) on prebiopsy orbital CT scans.

\begin{tabular}{|c|c|c|c|}
\hline Orbital CT Scans & $\begin{array}{c}\text { Orbital Lymphoma } \\
n(\%)\end{array}$ & $\begin{array}{c}\text { IgG4-ROD } \\
n(\%)\end{array}$ & $p$ \\
\hline Tumor number & & & $0.0414^{\#}$ \\
\hline Single & $11(85)$ & $2(15)$ & \\
\hline Multiple $(\geq 2)$ & $5(42)$ & $7(58)$ & \\
\hline Orbital involvement & & & $0.0168^{\#}$ \\
\hline One side & $12(86)$ & $2(14)$ & \\
\hline Bilateral & $4(36)$ & $7(64)$ & \\
\hline Bilateral lacrimal gland involvement & & & 0.0022 \# \\
\hline Presence & $2(22)$ & $7(78)$ & \\
\hline Absence & $14(88)$ & $2(12)$ & \\
\hline Sinusitis & & & 0.6882 \# \\
\hline Presence & $7(58)$ & $5(42)$ & \\
\hline Absence & $9(69)$ & $4(31)$ & \\
\hline
\end{tabular}


Specifically, of the 16 patients with OL, eleven (69\%) had a solitary tumor in an orbital cavity or at eyelids, $1(6 \%)$ had 2 tumors, $2(13 \%)$ had 3 and $2(13 \%)$ had 4 . The other 9 out of the 25 patients had IgG4-ROD: 2 (22\%) with 1 tumor; 5 (56\%) with 2 tumors, 1 (11\%) with 3 tumors and 1 (11\%) with 5 tumors. CT features statistically significantly associated with IgG4-ROD included lesions with regular borders $(p=0.0069)$, multiple tumors $(p=0.0414)$, lacrimal gland involvement $(p=0.0085)$, lesions involving bilateral lacrimal glands and bilateral orbital cavities $(p=0.0022$ and $p=0.0168$, respectively, Figure 1).

In contrast, tumors involving the extraconal, conal or intraconal space, lacrimal sac, optic nerve, extraocular muscle tendon, infraorbital nerve, preseptal space and presence of sinusitis and bone remodeling were ineffectual to differentiate IgG4-ROD from orbital lymphoma (Figures 1 and 2; all $p>0.05$, Fisher's exact test). Two IgG4-ROD patients and $6 \mathrm{OL}$ patients had a solitary tumor involving the preseptal space (Figure 2).

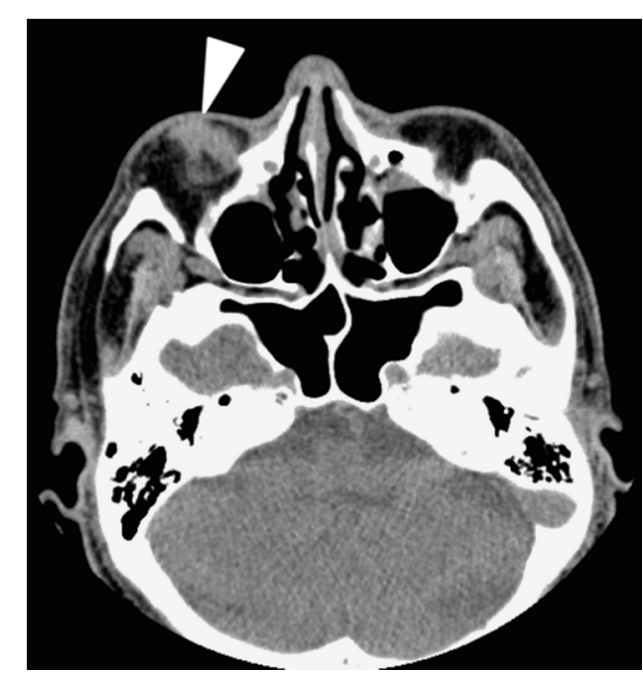

(a)

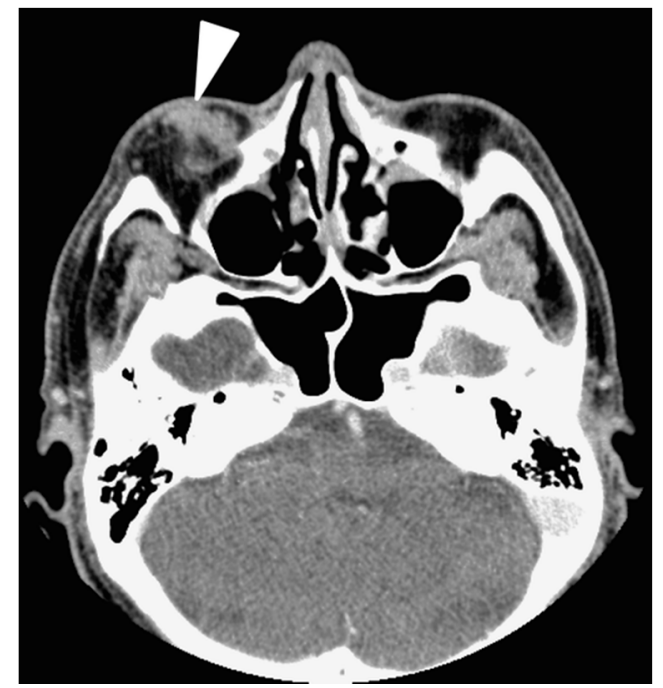

(b)

Figure 2. A 66-year-old man with orbital lymphoma shows a painless solitary lesion at the right lower eyelid. (a) Axial precontrast CT image shows a small nodule with an irregular border and homogeneous density involves the preseptal space of the right lower eyelid (arrowhead). Precontrast CT Hounsfield unit scale (CTHU) of the nodule is 57; (b) Axial postcontrast CT image shows the nodule demonstrates homogeneous enhancement (arrowhead). Postcontrast CTHU of the nodule is 68. Serum IgG4 level of the patient is $44.3 \mathrm{mg} / \mathrm{dL}$.

Table 3 shows the descriptive statistical prebiopsy values of precontrast CT Hounsfield unit scales (CTHU), postcontrast CTHU and postcontrast CTHU/precontrast CTHU ratios of 27 tumors of OL and 20 tumors of IgG4-ROD on prebiopsy CT scans.

Table 4 demonstrates descriptive statistical prebiopsy values of serum IgG4 levels, serum IgG levels and the ratios of serum IgG4 level/serum IgG level of the 16 patients with OL and the 9 patients with IgG4-ROD. 
Table 3. Descriptive statistical prebiopsy values of precontrast CT Hounsfield unit scales (Pre HU), postcontrast CTHU (Post HU) and postcontrast CTHU/precontrast CTHU ratios (Post HU/Pre HU) of 27 tumors of orbital lymphoma and 20 tumors of IgG4-related orbital disease (IgG4-ROD) on prebiopsy orbital CT scans.

\begin{tabular}{|c|c|c|c|c|c|c|}
\hline \multirow[b]{2}{*}{ Parameter } & \multicolumn{3}{|c|}{$\begin{array}{l}\text { Orbital Lymphoma } \\
\qquad n=27\end{array}$} & \multicolumn{3}{|c|}{$\begin{array}{c}\text { IgG4-ROD } \\
n=20\end{array}$} \\
\hline & Pre HU & Post HU & Post HU/Pre HU & Pre HU & Post HU & Post HU/Pre HU \\
\hline Median & 49 & 80 & 1.51 & 51 & 93.5 & 1.8 \\
\hline Mean & 51.8 & 78.9 & 1.55 & 54.1 & 93.8 & 1.77 \\
\hline SD & 10.28 & 13.58 & 0.27 & 10.69 & 19.02 & 0.42 \\
\hline Quartile 1 & 45 & 68 & 1.37 & 45.5 & 87 & 1.41 \\
\hline Quartile 3 & 57 & 89 & 1.76 & 61.25 & 103 & 2.08 \\
\hline minimum & 36 & 56 & 1.17 & 40 & 51 & 1.06 \\
\hline Maximum & 76 & 102 & 2.31 & 77 & 125 & 2.49 \\
\hline Outlier 1 & 76 & & 2.31 & & 51 & \\
\hline Outlier 2 & & & & & 59 & \\
\hline
\end{tabular}

n—number; SD—standard deviation.

Table 4. The descriptive statistical prebiopsy values of serum IgG4 levels (serum $\operatorname{IgG} 4$ ), serum immunoglobulin $\mathrm{G}(\mathrm{IgG})$ levels (serum $\operatorname{IgG}$ ) and the ratios of serum IgG4 level/serum IgG level (serum IgG4/IgG) of the 16 patients with orbital lymphoma and the 9 patients with IgG4-ROD.

\begin{tabular}{|c|c|c|c|c|c|c|}
\hline \multirow[b]{2}{*}{$\begin{array}{l}\text { Parameter } \\
(\mathrm{mg} / \mathrm{dL})\end{array}$} & \multicolumn{3}{|c|}{$\begin{array}{c}\text { Orbital Lymphoma } \\
\qquad n=16\end{array}$} & \multicolumn{3}{|c|}{$\begin{array}{c}\text { IgG4-ROD } \\
\quad n=9\end{array}$} \\
\hline & Serum IgG4 & Serum IgG & Serum IgG4/IgG & Serum IgG4 & Serum IgG & Serum IgG4/IgG \\
\hline Median & 57.65 & 1345 & 0.042 & 675 & 1560 & 0.241 \\
\hline Mean & 135.73 & 1307.94 & 0.106 & 756.49 & 2108.56 & 0.32 \\
\hline SD & 199.82 & 332.42 & 0.1502 & 733.78 & 1326.07 & 0.2325 \\
\hline Quartile 1 & 28.23 & 979 & 0.022 & 142 & 1232 & 0.107 \\
\hline Quartile 3 & 125.4 & 1594.5 & 0.123 & 1140 & 3080 & 0.563 \\
\hline minimum & 8.1 & 725 & 0.011 & 28.9 & 1110 & 0.026 \\
\hline Maximum & 780 & 1915 & 0.524 & 2297.9 & 4645 & 0.59 \\
\hline Outlier 1 & 339.5 & & 0.404 & & 4112 & \\
\hline Outlier 2 & 780 & & 0.524 & & 4645 & \\
\hline
\end{tabular}

Figures 3 and 4 show the differences in postcontrast CTHU, postcontrast CTHU/precontrast CTHU ratios, serum IgG4 levels and serum IgG4 level/serum IgG level ratios were statistically significant between the two groups (all $p<0.05$, Mann-Whitney U test).

Figure 3 shows that the areas under the ROC curve (AUC) of precontrast CTHU, postcontrast CTHU and the ratios of postcontrast CTHU/precontrast CTHU were 0.56 (95\% CI: $0.393-0.727, p=0.484$ ), 0.766 (95\% CI: 0.615-0.917, $p=0.002$ ) and 0.670 (95\% CI: 0.498-0.842, $p=0.048$ ). According to Figure 4, the AUC for serum IgG4 levels, serum IgG levels and the ratios of serum IgG4/serum IgG were 0.847 (95\% CI: 0.674-1.000, $p=0.005$ ), 0.684 (95\% CI: 0.455-0.913, $p=0.134)$ and 0.819 (95\% CI: 0.639-1.000, $p=0.009)$, respectively.

Compared with above data, the AUC using postcontrast CTHU (=0.766) and serum IgG4 levels $(=0.847)$ was moderately accurate for the diagnostic yield of IgG4-ROD because both AUC measures fell between 0.7 and 0.9 . The largest Jordon index 0.528 [(sensitivity-[1-specificity] $=0.528)]$ suggested a cutoff value of postcontrast CTHU equal to 89.5 . The sensitivity and specificity were 0.75 ( $95 \% \mathrm{CI}$ : 0.509-0.913) and 0.778 (95\% CI: 0.578-0.914), respectively. As to serum IgG4 level, the largest Jordon index (0.701) suggested a cutoff value equal to $132.5 \mathrm{mg} / \mathrm{dL}$, which resulted in sensitivity of 0.889 (95\% CI: 0.518-0.997) and specificity of 0.813 (95\% CI: 0.544-0.960), respectively. 
For patients with postcontrast CTHU $\geq 89.5$ in at least one orbital nodule in the two groups, 7 (58\%) were IgG4-ROD patients and $5(42 \%)$ were OL patients ( $p=0.0414$, Fisher's exact test). For serum IgG4 levels $\geq 132.5 \mathrm{mg} / \mathrm{dL}, 8(73 \%)$ were IgG4-ROD patients and $3(27 \%)$ OL $(p=0.0021)$.

The postcontrast CTHU and serum IgG4 levels for patients with a solitary orbital tumor in the two groups of OL and IgG4-ROD patients were as follows: higher postcontrast CTHU $(\geq 89.5)$ in $3 \mathrm{OL}$ patients and $1 \mathrm{IgG} 4-\mathrm{ROD}$ case; lower postcontrast CTHU $(<89.5)$ in 8 OL and $1 \mathrm{IgG} 4-\mathrm{ROD}$; and lower serum IgG4 levels $(<132.5)$ in all 11 OL patients and 1 IgG4-ROD case with higher CTHU. In addition, one IgG4-ROD patient with a lower postcontrast CTHU showed a higher serum IgG4 level $\geq 132.5$.

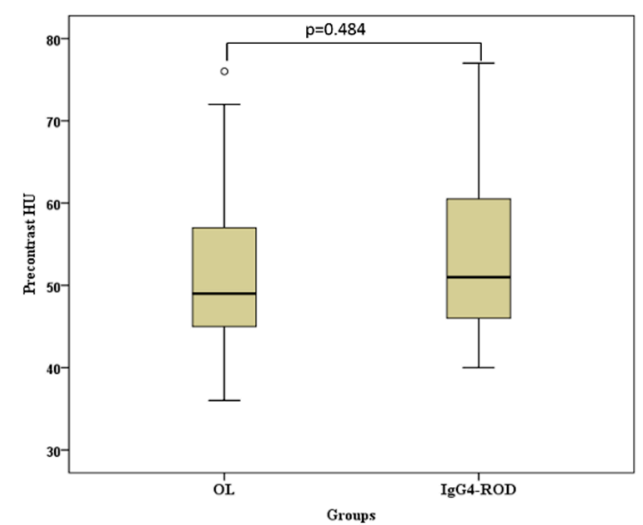

(a)

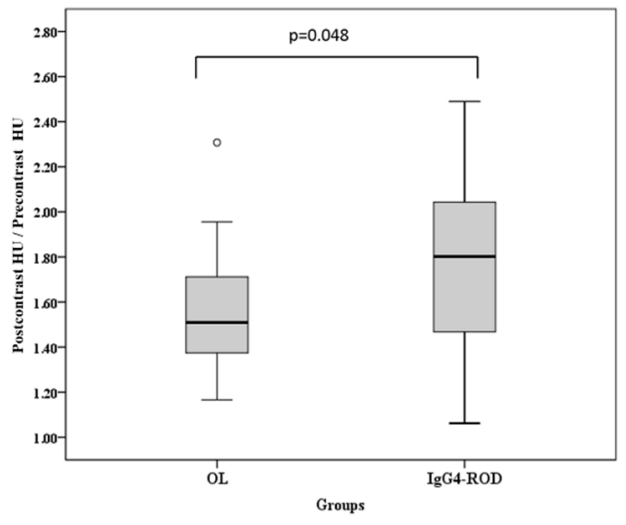

(c)

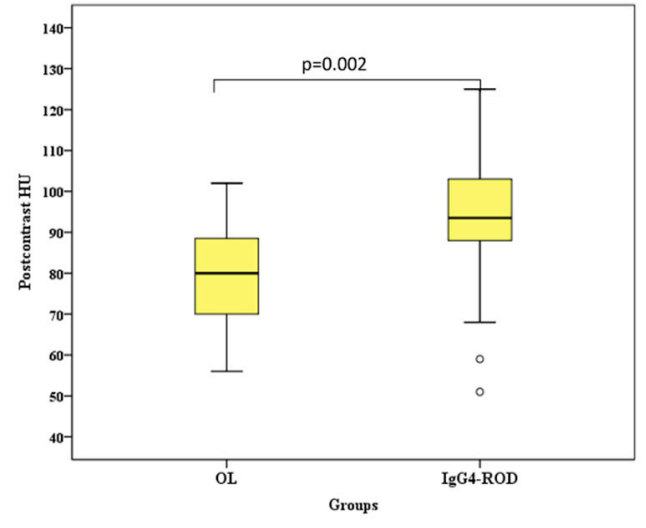

(b)

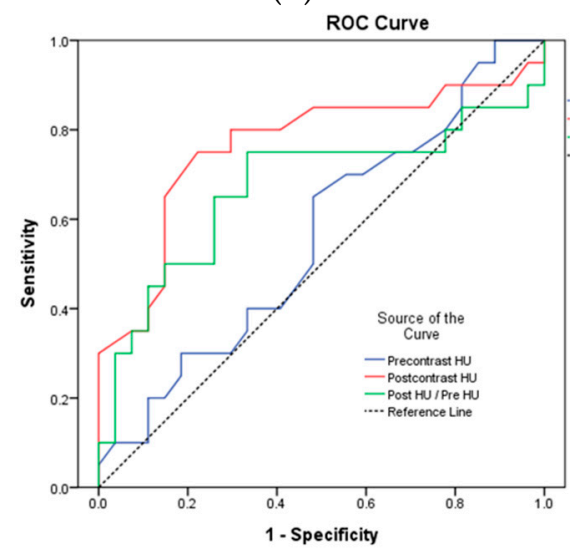

(d)

Figure 3. Box-and-whisker plots and receiver operating characteristic (ROC) curve analysis of precontrast computed tomography (CT) Hounsfield unit scale (CTHU), postcontrast CTHU, postcontrast CTHU/precontrast CTHU ratios between orbital lymphoma (OL) and immunoglobulin G4-related orbital disease (IgG4-ROD). Bars = medians. (a) Precontrast CTHU (precontrast HU) shows a nonsignificant difference between the two groups ( $p=0.484$, Mann-Whitney U test); (b) Postcontrast CTHU (postcontrast HU) shows a significant difference between the two groups ( $p=0.002$, Mann-Whitney U test); (c) Postcontrast CTHU/precontrast CTHU ratios (postcontrast HU/precontrast HU) show a significant difference between the two groups ( $p=0.048$, Mann-Whitney $U$ test); (d) Areas under the ROC curve (AUC) of precontrast CTHU (precontrast HU), postcontast CTHU (postcontrast HU) and postcontrast CTHU/precontrast CTHU ratios (postcontrast HU/precontrast HU) are 0.56 (95\% CI: 0.393-0.727, $p=0.484), 0.766$ (95\% CI: 0.615-0.917, $p=0.002)$ and 0.670 (95\% CI: $0.498-0.842, p=0.048$ ), respectively. $\_$-an outlier. 


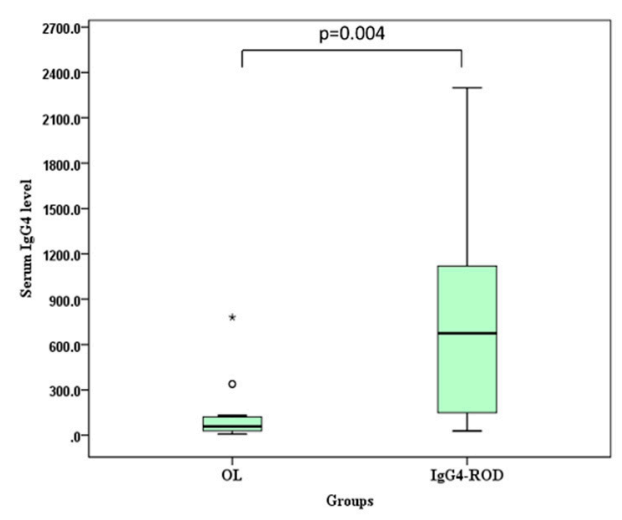

(a)

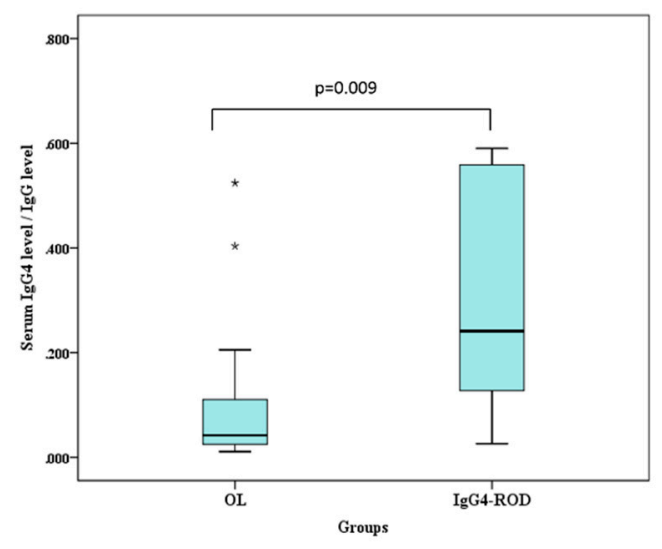

(c)

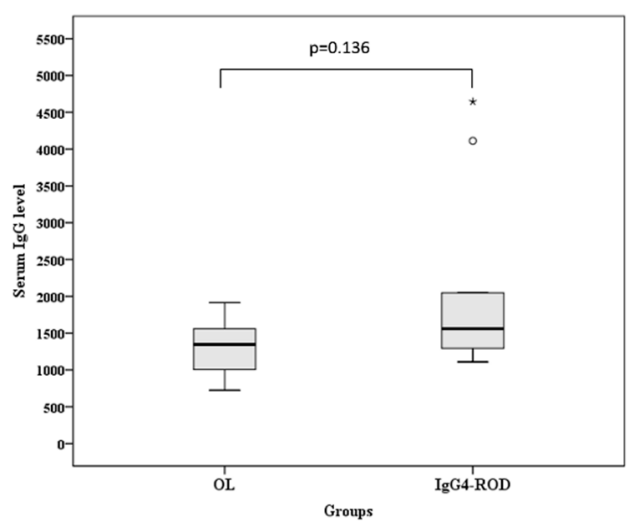

(b)

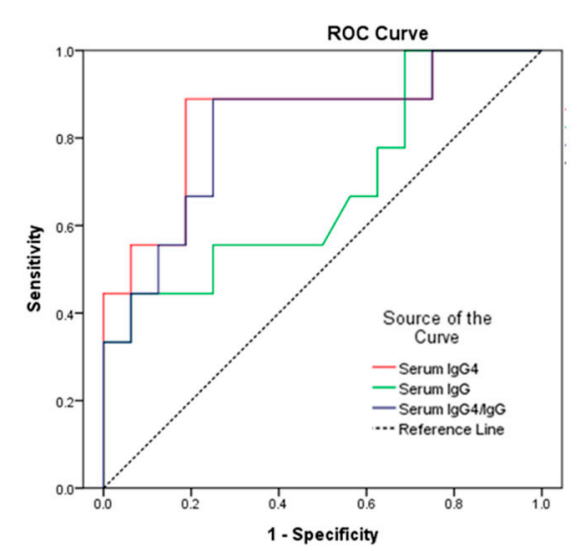

(d)

Figure 4. Box-and-whisker plots and receiver operating characteristic curve (ROC) analysis of serum immunoglobulin G4 (IgG4) levels, serum immunoglobulin G (IgG) levels and serum IgG4 level/serum IgG level ratios between orbital lymphoma (OL) and IgG4-related orbital disease (IgG4-ROD). Bars = medians. (a) Serum IgG4-levels demonstrate a significant difference between the two groups ( $p=0.004$, Mann-Whitney U test); (b) Serum IgG levels demonstrate a nonsignificant difference between the two groups ( $p=0.136$, Mann-Whitney U test); (c) Serum IgG4 level/IgG level ratios (serum IgG4 level/IgG level) demonstrate a significant difference between the two groups ( $p=0.009$, Mann-Whitney U test); (d) The areas under the ROC curve of serum IgG4 level (serum IgG4), serum IgG level (serum IgG) and serum IgG4 level/IgG level ratio (serum IgG4/IgG) are 0.847 (95\% CI: 0.674 to $1.000, p=0.005), 0.684$ (95\% CI: 0.455 to $0.913, p=0.134$ ) and 0.819 (95\% CI: 0.639 to $1.000, p=0.009$ ), respectively. ${ }^{*}$ and $\_$outliers.

The postcontrast CTHU and serum IgG4 level for patients with multiple orbital lesions in the two groups were as follows: lower postcontrast CTHU $(<89.5)$ were noted in $3 \mathrm{OL}$ patients and in 1 IgG4-ROD case; higher postcontrast CTHU $(\geq 89.5)$ were found in 2 OL patients and in 6 IgG4-ROD cases; higher serum IgG4 levels $(\geq 132.5 \mathrm{mg} / \mathrm{dL})$ were found in 3 OL patients and in 7 IgG4-ROD cases; lower serum IgG4 levels $(<132.5 \mathrm{mg} / \mathrm{dL})$ appeared in 2 OL patients. Two of the three OL patients with lower postcontrast CTHU showed OL involving bilateral lacrimal glands, who had different serum IgG4 levels: 51.3 and 339.5, respectively. Concurrent higher postcontrast CTHU and a higher serum IgG4 level were found in 1 OL patient $(1 / 5,20 \%)$ with multiple tumors in the left orbital cavity and in 6 IgG4-ROD cases with tumors involving bilateral lacrimal glands. Lower postcontrast CTHU and higher serum IgG4 level were noted in only one IgG4-ROD (1/7, 14\%) case, who showed tumors mainly in intraconal spaces of bilateral orbits.

If this study used "lesions with bilateral lacrimal gland involvement" (the most significant qualitative CT feature in statistics, $p=0.0022$ ), "bilateral lacrimal gland involvement and a higher 
serum IgG4 level $(\geq 132.5 \mathrm{mg} / \mathrm{dL})(p=0.0005)$ " or "bilateral lacrimal gland involvement and higher postcontrast CTHU ( $\geq 89.5$, quantitative CT feature) $(p=0.00047)$ " or "higher postcontrast CTHU and a higher serum IgG4 level" ( $p=0.0029$ ) as helpful test tools for diagnosis of IgG4-ROD (Table 5), sensitivity, specificity and accuracy of the first test (Test 1), the second (Test 2), the third (Test 3 ) and the latest one (Test 4) were as follows (Table 5): 0.78 (95\% CI: 0.3999-0.972), 0.88 (95\% CI: 0.617-0.985) and 0.84 (95\% CI: $0.639-0.955)$ for Test 1; 0.78 (95\% CI: 0.3999-0.972), 0.94 (95\% CI: 0.698-0.998) and 0.88 (95\% CI: $0.688-0.975)$ for Test 2; 0.67 (95\% CI: 0.299-0.925), 1 (95\% CI: 0.794-1) and 0.88 (95\% CI: 0.688-0.975) for Test 3; 0.67 (95\% CI: 0.299-0.925), 0.94 (95\% CI: 0.698-0.998) and 0.84 (95\% CI: $0.639-0.955)$ for Test 4 . In Table 5, Test 3 had $100 \%$ of positive predictive value (PPV). Test $1 \&$ Test 2 had the highest negative predictive value (NPV) 0.88 .

Table 5. Contingency table of four helpful testing tools for diagnosis of IgG4-related orbital disease (IgG4-ROD).

\begin{tabular}{ccccc}
\hline & Test 1 & Test 2 & Test 3 & Test 4 \\
\hline True positive, $n$ & 7 & 7 & 6 & 6 \\
False negative, $n$ & 2 & 2 & 3 & 3 \\
False positive, $n$ & 2 & 1 & 0 & 1 \\
True negative, $n$ & 14 & 15 & 16 & 15 \\
Sensitivity & 0.78 & 0.78 & 0.67 & 0.67 \\
(95\% CI) & $(0.40-0.972)$ & $(0.40-0.972)$ & $(0.299-0.925)$ & $(0.299-0.925)$ \\
Specificity & 0.88 & 0.94 & 1.0 & 0.94 \\
(95\% CI) & $(0.617-0.985)$ & $(0.698-0.998)$ & $(0.794-1.0)$ & $(0.698-0.998)$ \\
PPV & 0.78 & 0.88 & 1.0 & 0.86 \\
$(95 \%$ CI) & $(0.478-0.931)$ & $(0.504-0.98)$ & $(*)$ & $(0.460-0.977)$ \\
NPV & 0.88 & 0.88 & 0.84 & 0.83 \\
$(95 \%$ CI) & $(0.670-0.960)$ & $(0.687-0.962)$ & $(0.679-0.931)$ & $(0.663-0.927)$ \\
Accuracy & 0.84 & 0.88 & 0.88 & 0.84 \\
$(95 \%$ CI) & $(0.639-0.955)$ & $(0.688-0.975)$ & $(0.688-0.975)$ & $(0.639-0.955)$ \\
\hline
\end{tabular}

n, patient number; Test 1 to Test 4 represent four helpful tools for diagnosis of IgG4-ROD; Test 1, orbital lesions with bilateral lacrimal gland involvement on CT scans; Test 2, orbital lesions with bilateral lacrimal gland involvement on CT scans and a higher serum IgG4 level ( $\geq 132.5 \mathrm{mg} / \mathrm{dL}$ [milligrams per deciliter]); Test 3 , orbital lesions with bilateral lacrimal gland involvement and higher postcontrast CTHU (CT Hounsfield unit scales $\geq 89.5$ ) on CT scans; Test 4, orbital lesions with higher postcontrast CTHU $(\geq 89.5)$ and a higher serum IgG4 level $(\geq 132.5 \mathrm{mg} / \mathrm{dL}) ;(95 \% \mathrm{CI})$, ( $95 \%$ confidence interval); ${ }^{*}$ - not shown in the statistics operation; PPV—-positive predictive value-NPV—negative predictive value.

\section{Discussion}

The IgG4-related disease can result in fibroinflammatory lesions at nearly any anatomic site [14]. OL is malignant and needs radiotherapy, chemotherapy and/or operation $[4,7,8]$. IgG4-ROD is benign and approximately $90 \%$ of patients respond well to steroid treatment [5]. Both of malignant OL and benign IgG4-ROD are lymphoplasmacytic infiltrated mass-like lesions, which make clinicians difficult to differentiate from each other [6]. This study showed that lesions with regular borders, multiple tumors, lacrimal gland involvement, simultaneous involvement of bilateral lacrimal glands and bilateral orbital cavities and higher medians of postcontrast CTHU and serum IgG4 levels were significantly related to IgG4-ROD (all $p<0.05$ ). Postcontrast CTHU $\geq 89.5$ showed 0.75 sensitivity and 0.778 specificity with the AUC $=0.766$ (95\% CI: $0.615-0.917, p=0.002$ ); serum IgG4 levels $\geq 132.5$ $\mathrm{mg} / \mathrm{dL}$ had 0.889 sensitivity and 0.813 specificity, with the AUC $=0.847$ (95\% CI: $0.674-1.000, p=0.005$, Figures 3 and 4). A lesion with regular borders is most likely to be a slow growing benign mass or less likely to be an indolent malignant tumor [1]. IgG4-ROD being benign usually presented as lesions with regular borders in this study.

Serum IgG4 levels account for 3\% to 6\% total amount of serum IgG levels [15]. Hamano et al. [16] reported a cutoff value of $135 \mathrm{mg} / \mathrm{dL}$ to differentiate autoimmune pancreatitis from pancreatic cancer with a high sensitivity (95\%), specificity $(97 \%)$ and accuracy $(97 \%)$. This study identified a cutoff value 
$132.5 \mathrm{mg} / \mathrm{dL}$ (close to $135 \mathrm{mg} / \mathrm{dL}$ ) to distinguish IgG4-ROD from OL at diagnostic accuracy (AUC) of $84.7 \%$ with $88.9 \%$ sensitivity and $81.3 \%$ specificity.

However, approximately $40-50 \%$ of patients with biopsy-proven IgG4-related disease have normal serum IgG4 concentrations $[10,14,17]$. In our study, normal serum IgG4 $(<132.5 \mathrm{mg} / \mathrm{dL})$ occurred in $13(81 \%)$ of 16 OL patients and one IgG4-ROD patient $(1 / 9,11 \%)$, who had a solitary orbital lesion. There may be several reasons to explain why in our study there was a lower percentage of IgG4-ROD with normal serum IgG4 levels: first, our study was a small sample research, which may have selection bias; second, serum IgG4 levels may vary according to the specific organ involved [10]; finally, elevated serum IgG4 levels represent a subtype of IgG4-related disease with more inflammatory features and worsening disease activity [17]. Our IgG4-ROD patients $(8 / 9,89 \%)$ could be developing an active IgG4-related disease with elevated serum IgG4 concentrations.

Patient's age, standard imaging features and localizing orbital lesions to intraconal, conal or extraconal compartments help limit the differential diagnosis [18]. Our study showed no significant difference in median ages between patients with OL and IgG4-ROD ( $p=0.934$, Mann-Whitney $\mathrm{U}$ test). Lesions with regular borders, multiple orbital tumors, lacrimal gland involvement, lesions simultaneously involving bilateral lacrimal glands and bilateral orbital cavities and higher postcontrast CTHU ( $\geq 89.5$ ) on orbital CT scans were significantly associated with IgG4-ROD (all $p<0.05)$. The difference in extraconal, conal and intraconal compartments of orbital lesions between OL and IgG4-ROD groups was not statistically significant $(p=0.4813)$. In addition to $\mathrm{CT}$, magnetic resonance imaging (MRI) also helps in further diagnostic workup of orbital tumors and provides ocular anatomy for lesions involvement, perineural spread and intracranial extension [12]. Both retinoblastomas typically found in children and uveal melanomas in adults appear in the globe. Retinoblastoma is slightly hyperintense on T1 weighted MRI (T1WI) and very hypointense relative to vitreous on T2-weighted MRI (T2WI) and well contrast enhancement on postcontrast CT and contrast-enhanced (CE) MRI [12,18]. Ninety percent of retinoblastomas demonstrate calcifications on precontrast CT scans [18]. Melanomas with melanin show characteristic hyperintensity on T1WI and hypointensity on T2WI [12,18]. For intraconal orbital tumors, gliomas common among children result in fusiform enlargement of the optic nerve on axial CT and MRI [12,18]. In contrast, meningiomas, commonly seen in the 5th decade of life, classically show the contrast-enhancing tumor with a "tram-tract" configuration alongside the optic nerve on axial postcontrast CT or CE MRI [12]. The most common benign orbital tumor in adults is a cavernous hemangioma, which typically demonstrates a well-defined dense unilateral orbital intraconal mass with intra-tumoral calcifications on precontrast CT scans and MRI. The enhancement spread pattern on a dynamic postcontrast CT and dynamic CE T1WI can help to distinguish between cavernous hemangioma and schwannoma $[18,19]$. Cavernous hemangiomas show initial patchy enhancement on arterial phase, but schwannomas start a wide area of enhancement. The most common congenital orbital nodules are dermoids, which usually show a well outlined round or oval tumor with a capsule and low density or fat contents in the extraconal space on CT scans or MRI [18]. Due to fat contents, dermoids typically show hyperintensity on T1WI, hyperintensity on T2WI and hypointensity on short tau inversion recovery MRI (STIR) [18]. Benign mixed tumor of lacrimal gland usually seen in middle-aged patients demonstrates a well-circumscribed round or oval tumor with homogeneous enhancement on postcontrast CT and CE MRI [12]. Malignant epithelial lacrimal gland tumors show a mass with a well- or poor-defined margin with associated bony remodeling or destruction in $70 \%$ cases on CT scans [18].

Multiple or multicompartmental orbital masses include venolymphatic malformations (VLM), rhabdomyosarcoma (RMS), plexiform neurofibroma, thyroid ophthalmopathy (TO), orbital pseudotumor (OP), lymphoma, metastases and IgG4-ROD [18]. The first three types of masses are common among children; the last five, among adults [3,5,18]. VLM usually appears poorly defined, lobulated and multiloculated lesions with various signal intensity on T1WI and T2WI [18]. VLM may demonstrate fluid-fluid level on MRI, which is highly suggestive of the diagnosis of VLM [12]. VLM, RMS and plexiform neurofibroma may have similar findings on CT and MRI [18]. TO causes enlarged 
bilateral myositis of the extraocular muscles, often involves medial and inferior rectus muscles with sparing tendinous insertions on CT and MRI [12] and is related to elevated thyroid-stimulating hormone level [18]. OP, IgG4-ROD and OL show similar MRI features on conventional sequences, which are hypointense on T1WI and T2WI and well contrast enhancement on postcontrast T1WI. Furthermore, diffusion-weighted imaging (DWI) with apparent diffusion coefficient (ADC) mapping can help to differentiate between benign and malignant orbital lesions [1,18]. Sepahdari et al. have reported that an ADC value $<1.0 \times 10^{-3} \mathrm{~mm}^{2} / \mathrm{sec}$ and an ADC ratio $<1.2$ are optimal for predicting orbital malignant tumors [1,18]. Prior studies used ADC value $<1.0 \times 10^{-3} \mathrm{~mm}^{2} / \mathrm{sec}$ and ADC ratio $<1.2$ to differentiate orbital lymphoma from benign OP and IgG4-ROD with more than 95\% accuracy $[1,18]$. However, ADC values and ratios cannot differentiate OP from IgG4-ROD because the two disease have similar these values $[1,18]$. OP manifests with the most common acute unilateral painful mass in adults, which assist in differentiating OP from TO, OL and IgG4-ROD. Pain is uncommon in TO, OL and IgG4-ROD [12,18]. None of 25 patients in this study suffered from orbital pain or tender palpable mass, either. Consistent with the result of our research, Fujita et al. have reported that IgG4-ROD commonly presents involving bilateral lacrimal glands [15], which can distinguish OL and OP from IgG4-ROD. A clinician can suggest the diagnosis of orbital metastasis only when clinically primary malignancy is known [18].

An effective clinical diagnosis or appropriate disease classification for IgG4-related disease needs the integration of clinical findings, radiological features and serologic or pathologic data [12]. None of our 25 patients suffered from painful orbital lesions. Four tests for IgG4-ROD diagnosis used in this study included serologic serum IgG4 levels, radiological CT qualitative (lesions with bilateral lacrimal gland involvement) and/or quantitative features (postcontrast CTHU $\geq 89.5$ ). Of the four tests, Test 2 (lesions with bilateral lacrimal gland involvement and a higher serum IgG4 level [ $\geq 132.5 \mathrm{mg} / \mathrm{dL}]$ ) with the highest sensitivity (78\%), a higher specificity $(94 \%)$, a higher PPV $(88 \%)$, the highest NPV $(88 \%)$ and the highest accuracy $(88 \%)$ could be the better prebiopsy test to distinguish IgG4-ROD from OL.

This study had only two IgG4-ROD patients with a solitary tumor, which was a small sample and lacked specific CT features. Prebiopsy diagnosis of a solitary IgG4-ROD could depend on a painless orbital mass, postcontrast CTHU $\geq 89.5$ and serum IgG4 level $\geq 132.5 \mathrm{mg} / \mathrm{dL}$. Tissue proof is an ultimate diagnostic way. However, biopsy is not always suitable for orbital lesions. The best medical option for a benign mixed tumor or malignant mass of the lacrimal gland may be excision en bloc without biopsy once clinical and imaging diagnosis. However, incomplete excision or ruptures of neoplasms via biopsy may result in tumor recurrence, malignant transformation of a mixed tumor and poor prognosis [12]. In clinical practice, some IgG4-ROD patients may be at high risk for biopsy and/or refuse biopsy. However, once these patients meet possible diagnosis of IgG4-ROD [20], systemic steroid treatment may be a good alternative. The criteria for possible IgG4-ROD diagnosis include elevated serum IgG4 ( $\geq 135 \mathrm{mg} / \mathrm{dL}$ ), enlargement of the lacrimal gland or masses, enlargement or hypertrophic lesions in various orbital tissues [20]. Clinicians could forgo further biopsy if such patients respond well to glucocorticoids within weeks, such as reductions in the size of tumors, improvements of symptoms and a significant decrease in serum IgG4 [20]. Alternative non-vital organ or lip biopsy may be an acceptable option [21].

Sato et al. [22] reported that 17 (81\%) of 21 patients with IgG4-ROD had involvement of the lacrimal glands and $13(70.6 \%)$ of 17 cases showed bilateral lacrimal gland swellings. Our study also showed that seven $(78 \%)$ of nine patients with IgG4-ROD had bilateral lacrimal gland involvement, which could distinguish IgG4-ROD from OL $(p=0.0022)$. Neither Sato et al. nor this study had patients with IgG4-ROD originating from conjunctival or subconjunctival tissue. However, prior research suggested that IgG4-ROD can also develop at conjunctival tissue [23,24].

This study has several limitations. First, this retrospective research had a small sample of patients. Second, the patients in this study received different brands of contrast agents and CT machine, which could produce potential bias in measurement of CTHU. Last, incomplete data records ruled out the 
possibility of deciphering a detailed correlation between $\mathrm{CT}$ features and clinical presentations of OL and IgG4 ROD.

\section{Conclusions}

This study compared prebiopsy precontrast and postcontrast CT features, serum IgG4 and serum IgG levels of IgG4-ROD with those of OL. The key findings showed that IgG4-ROD had high correlation with the presence of lesions with regular borders, presence of multiple lesions, lesions involving the lacrimal gland, both lacrimal glands and bilateral orbital cavities on CT scans, higher values of postcontrast CTHU, postcontrast CTHU/precontrast CTHU ratios, serum IgG4 levels and serum IgG4/IgG level ratios (all $p<0.05$ ). For diagnosis of IgG4-ROD, postcontrast CTHU $\geq 89.5$ and serum IgG4 level $\geq 132.5 \mathrm{mg} / \mathrm{dL}$ provided moderate diagnostic accuracy, AUC $=0.776$ and 0.847 , respectively, which were higher than those of postcontrast HU/precontrast HU and serum IgG4/IgG level ratio. The special CT features and elevated serum IgG4 levels could help differentiate IgG4-ROD from OL. Prebiopsy diagnosis of the uncommon solitary type of IgG4-ROD could also depend on a painless orbital mass and elevated CTHU $\geq 89.5$ and serum IgG4 level $\geq 132.5 \mathrm{mg} / \mathrm{dL}$.

Author Contributions: Conceptualization, W.-H.Y. and A.F.-Y.L.; methodology, W.-H.Y. and A.F.-Y.L.; software, W.-H.Y.; validation, W.-H.Y. and A.F.-Y.L.; formal analysis, Y.-Y.C., C.-H.W., H.-C.H., A.F.-Y.L.; investigation, Y.-Y.C., C.-H.W., H.-C.H., A.F.-Y.L.; resources, S.-Y.Y.; data curation, S.-Y.Y., W.-H.Y.; writing-original draft preparation, W.-H.Y.; writing-review and editing, W.-H.Y.; visualization, W.-Y.G.; supervision, J.-F.L. and W.-Y.G.; project administration, W.-H.Y.; funding acquisition, W.-H.Y. All authors have read and agreed to the published version of the manuscript.

Funding: This research received no external funding.

Acknowledgments: We thank May Yuan for English language editing.

Conflicts of Interest: The authors declare no conflict of interests.

\section{References}

1. Sepahdari, A.R.; Aakalu, V.K.; Setabutr, P.; Shiehmorteza, M.; Naheedy, J.H.; Mafee, M.F. Indeterminate orbital masses: Restricted diffusion at MR imaging with echo-planar diffusion-weighted imaging predicts malignancy. Radiology 2010, 256, 554-564. [CrossRef]

2. Shields, J.A.; Shields, C.L.; Scartozzi, R. Survey of 1264 patients with orbital tumors and simulating lesions: The 2002 Montgomery Lecture, part 1. Ophthalmology 2004, 111, 997-1008. [CrossRef]

3. Politi, L.S.; Forghani, R.; Godi, C.; Resti, A.G.; Ponzoni, M.; Bianchi, S.; Iadanza, A.; Ambrosi, A.; Falini, A.; Ferreri, A.J.; et al. Ocular adnexal lymphoma: Diffusion-weighted $\mathrm{mr}$ imaging for differential diagnosis and therapeutic monitoring. Radiology 2010, 256, 565-574. [CrossRef] [PubMed]

4. Sjo, L.D. Ophthalmic lymphoma: Epidemiology and pathogenesis. Acta Ophthalmol. 2009, 87, 1-20. [CrossRef] [PubMed]

5. Detiger, S.E.; Karim, A.F.; Verdijk, R.M.; van Hagen, P.M.; van Laar, J.A.M.; Paridaens, D. The treatment outcomes in IgG4-related orbital disease: A systematic review of the literature. Acta Ophthalmol. 2019, 97, 451-459. [CrossRef]

6. Hiwatashi, A.; Yoshiura, T.; Togao, O.; Yamashita, K.; Kikuchi, K.; Fujita, Y.; Yoshikawa, H.; Koga, T.; Obara, M.; Honda, H. Diffusivity of intraorbital lymphoma vs. IgG4-related DISEASE: 3D turbo field echo with diffusion-sensitised driven-equilibrium preparation technique. Eur. Radiol. 2014, 24, 581-586.

7. Damato, B.; Bever, G.J.; Kim, D.J.; Afshar, A.R.; Rubenstein, J.L. An audit of retinal lymphoma treatment at the University of California San Francisco. Eye 2019, 34, 515-522. [CrossRef] [PubMed]

8. Lee, J.; Yoon, J.S.; Kim, J.S.; Koom, W.S.; Cho, J.; Suh, C.O. Long-term outcome, relapse patterns, and toxicity after radiotherapy for orbital mucosa-associated lymphoid tissue lymphoma: Implications for radiotherapy optimization. Jpn. J. Clin. Oncol. 2019, 49, 664-670. [CrossRef] [PubMed]

9. Toyoda, K.; Oba, H.; Kutomi, K.; Furui, S.; Oohara, A.; Mori, H.; Sakurai, K.; Tsuchiya, K.; Kan, S.; Numaguchi, Y. MR imaging of IgG4-related disease in the head and neck and brain. AJNR Am. J. Neuroradiol. 2012, 33, 2136-2139. [CrossRef] 
10. Ginat, D.T.; Freitag, S.K.; Kieff, D.; Grove, A.; Fay, A.; Cunnane, M.; Moonis, G. Radiographic patterns of orbital involvement in IgG4-related disease. Ophthalmic Plast. Reconstr. Surg. 2013, 29, 261-266. [CrossRef]

11. Khosroshahi, A.; Cheryk, L.A.; Carruthers, M.N.; Edwards, J.A.; Bloch, D.B.; Stone, J.H. Brief Report: Spuriously low serum IgG4 concentrations caused by the prozone phenomenon in patients with IgG4-related disease. Arthritis Rheumatol. 2014, 66, 213-217. [CrossRef] [PubMed]

12. Tailor, T.D.; Gupta, D.; Dalley, R.W.; Keene, C.D.; Anzai, Y. Orbital neoplasms in adults: Clinical, radiologic, and pathologic review. Radiographics 2013, 33, 1739-1758. [CrossRef] [PubMed]

13. Yuan, W.H.; Hsu, H.C.; Cheng, H.C.; Guo, W.Y.; Teng, M.M.; Chen, S.J.; Lin, T.C. CT of globe rupture: Analysis and frequency of findings. AJR Am. J. Roentgenol. 2014, 202, 1100-1107. [CrossRef] [PubMed]

14. Wallace, Z.S.; Naden, R.P.; Chari, S.; Choi, H.K. The 2019 American College of Rheumatology/European League Against Rheumatism classification criteria for IgG4-related disease. Arthritis Rheumatol. 2020, 72, 7-19. [CrossRef] [PubMed]

15. Fujita, A.; Sakai, O.; Chapman, M.N.; Sugimoto, H. IgG4-related disease of the head and neck: CT and MR imaging manifestations. Radiographics 2012, 32, 1945-1958. [CrossRef] [PubMed]

16. Hamano, H.; Kawa, S.; Horiuchi, A.; Unno, H.; Furuya, N.; Akamatsu, T.; Fukushima, M.; Nikaido, T.; Nakayama, K.; Usuda, N.; et al. High serum IgG4 concentrations in patients with sclerosing pancreatitis. N. Engl. J. Med. 2001, 344, 732-738. [CrossRef]

17. Wallace, Z.S.; Deshpande, V.; Mattoo, H.; Mahajan, V.S.; Kulikova, M.; Pillai, S.; Stone, J.H. IgG4-Related Disease: Clinical and Laboratory Features in One Hundred Twenty-Five Patients. Arthritis Rheumatol. 2015, 67, 2466-2475. [CrossRef]

18. Purohit, B.S.; Vargas, M.I.; Ailianou, A.; Merlini, L.; Poletti, P.A.; Platon, A.; Delattre, B.M.; Rager, O.; Burkhardt, K.; Becker, M. Orbital tumours and tumour-like lesions: Exploring the armamentarium of multiparametric imaging. Insights Imaging 2016, 7, 43-68. [CrossRef]

19. Tanaka, A.; Mihara, F.; Yoshiura, T.; Togao, O.; Kuwabara, Y.; Natori, Y.; Sasaki, T.; Honda, H. Differentiation of cavernous hemangioma from schwannoma of the orbit: A dynamic MRI study. AJR Am. J. Roentgenol. 2004, 183, 1799-1804. [CrossRef]

20. Yu, W.K.; Tsai, C.C.; Kao, S.C.; Liu, C.J. Immunoglobulin G4-related ophthalmic disease. Taiwan J. Ophthalmol. 2018, 8, 9-14. [CrossRef]

21. Akiyama, M.; Kaneko, Y.; Hayashi, Y.; Takeuchi, T. IgG4-related disease involving vital organs diagnosed with lip biopsy: A case report and literature review. Medicine 2016, 95, e3970. [CrossRef] [PubMed]

22. Sato, Y.; Ohshima, K.; Ichimura, K.; Sato, M.; Yamadori, I.; Tanaka, T.; Takata, K.; Morito, T.; Kondo, E.; Yoshino, T. Ocular adnexal IgG4-related disease has uniform clinicopathology. Pathol. Int. 2008, 58, 465-470. [CrossRef] [PubMed]

23. da Fonseca, F.L.; Ramos Rde, I.; de Lima, P.P.; Nogueira, A.B.; Matayoshi, S. Unilateral eyelid mass as an unusual presentation of ocular adnexal IgG4-related inflammation. Cornea 2013, 32, 517-519. [CrossRef] [PubMed]

24. Lee, H.S.; Choi, W.; Kim, G.E.; Yoon, K.C. Case of Primary Isolated Subconjunctival IgG4-Related Disease. Cornea 2018, 37, 926-928. [CrossRef] [PubMed]

(C) 2020 by the authors. Licensee MDPI, Basel, Switzerland. This article is an open access article distributed under the terms and conditions of the Creative Commons Attribution (CC BY) license (http://creativecommons.org/licenses/by/4.0/). 